\title{
Le baccalauréat international
}

Parcours complexe d'un projet, de Sèvres à l'Équateur

The International Baccalaureate. The complex journey of a project, from Sèvres to Ecuador

El bachillerato internacional. La trayectoria compleja de un proyecto, de Sèvres al Ecuador

\section{Tristan Bunnell}

Traducteur : Sylvaine Herold

\section{CpenEdition} Journals

Édition électronique

URL : https://journals.openedition.org/ries/6055

DOI : $10.4000 /$ ries. 6055

ISSN : 2261-4265

Éditeur

France Education international

Édition imprimée

Date de publication : 1 décembre 2017

Pagination : 63-72

ISBN : 978-2-85420-616-6

ISSN : 1254-4590

Référence électronique

Tristan Bunnell, «Le baccalauréat international », Revue internationale d'éducation de Sèvres [En ligne],

76 | décembre 2017, mis en ligne le 01 décembre 2019, consulté le 25 juin 2021. URL : http://

journals.openedition.org/ries/6055 ; DOI : https://doi.org/10.4000/ries.6055 


\title{
Le baccalauréat international
}

Parcours complexe d'un projet, de Sèvres à l'Équateur*

\author{
Tristan Bunnell \\ University of Bath
}

\section{L'ÉMERGENCE DU BACCALAURÉAT INTERNATIONAL EN 1962}

Les origines du baccalauréat international (BI) de l'Organisation du baccalauréat international ${ }^{1}$, basée à Genève et dont la mission idéaliste singulière vise à «former des jeunes gens curieux, instruits et attentifs qui contribuent à créer un monde meilleur et plus pacifique par une meilleure compréhension et un plus grand respect interculturels ", paraissent de nos jours incroyablement modestes, en termes d'échelle comme d'ambition. Le programme originel du diplôme du $\mathrm{BI}^{2}$, en tant qu' « examen d'entrée international à l'université pouvant être passé et reconnu dans n’importe quel pays »(Peterson, 1972), a été débattu pour la première fois en 1962, lors d'une conférence d'enseignants en sciences sociales. Le BI a d'abord vu le jour comme programme d'histoire contemporaine, faisant l'objet d'un premier examen en juin 1963 à l'École internationale de Genève, auprès de cinq élèves seulement (Hill, 2010). Ce programme était " destiné à répondre aux besoins et aux aspirations des écoles internationales » (Peterson, 1972) ; l'un de ces élèves a notamment été accepté à Harvard par la suite (Hill, 2010). Puis, de 1964 à 1968, le BI a commencé à opérer sous la dénomination, pouvant prêter à confusion, de Service d'examen des écoles internationales ${ }^{3}$, avant de finalement s'établir sous le nom d' "Organisation du BI». Dès le départ, l'organisation "a employé l'anglais et le français comme langues de travail » et a bénéficié du soutien du ministère français de l'éducation (Peterson, 1972).

L'histoire de la création du BI met en lumière ses principaux fondateurs et soutiens, ainsi que le cours expérimental de son développement initial. Le besoin pragmatique d'un nombre croissant d' "écoles internationales " d'élite,

\footnotetext{
* Article traduit par Sylvaine Herold.

1. International Baccalaureate Office (IBO) en anglais. ( $N d T)$

2. IB Diploma Programme (IBDP) en anglais. ( $N d T$ )

3. International Schools Examination Syndicate (ISES) en anglais. (NdT)
} 
afin d'accueillir les enfants des cadres du corps diplomatique et des compagnies multinationales, est au fondement même du projet. De même, les sciences sociales, et notamment l'histoire, sont au cœur de son idéal irénique. L'Europe du Nord en est le centre d'activité principal et Genève "l'endroit où le diplôme du BI a été conçu » (Hill, 2010). Ce scénario initial diffère singulièrement de la situation actuelle, qui sera examinée dans cet article, et dans laquelle le BI joue un rôle de plus en plus important dans l'éducation des jeunes des écoles publiques, que ce soit à Adelaïde, à Chicago, au Pérou ou encore en Équateur.

Cela étant dit, cette "expérience d'éducation internationale » (Peterson, 1972) a démarré avec une portée géographique extrêmement modeste. En juin 1967, seulement 147 étudiants ont passé les examens expérimentaux en histoire et en géographie, à l'École internationale de Genève et à l'Atlantic College, au sud du Pays de Galles. En mai 1968, cinq nouvelles écoles étaient apparues (au Danemark, en Iran, en Angleterre, au Liban et aux États-Unis) et, au total, ces sept écoles ont inscrit 349 élèves dans un large éventail de matières. En 1969, 13 écoles, dont une en France, ont inscrit 720 élèves (Hill, 2010). Trois ans plus tard, l'un des principaux architectes de ce projet s'interrogeait humblement :

le projet de baccalauréat international est une expérience d'éducation internationale à petite échelle, conçue pour répondre aux problèmes limités auxquels sont actuellement confrontées les écoles internationales. Mais pourrait-il conduire à quelque chose de plus substantiel ? (Peterson, 1972)

Il notait cependant également des motifs de satisfaction :

il y a néanmoins déjà tout lieu de penser que cette expérience sera un succès et que le baccalauréat international deviendra un élément indispensable de l'offre éducative mondiale. (Peterson, 1972)

Les deux commentaires précédents de Peterson semblent de nos jours inutilement pessimistes. Au 16 mars 2017, il existait en effet 3104 écoles proposant le programme du diplôme du BI dans 147 pays. En fait, le «projet » de BI, qui comprend actuellement quatre programmes distincts, «a connu une évolution phénoménale au cours de ses quarante années d'existence, tant dans les services qu'il propose que dans son niveau d'influence» (Tarc, 2009). Au programme initial du diplôme du BI (l'IBDP $\left.{ }^{4}\right)$ se sont ajoutés : un programme primaire (PP) et un programme de premier cycle secondaire (PPCS), formant un « continuum complet d'études internationales »; il existe également un certificat à orientation professionnelle (COPIB), relativement récent. Au total, au 16 mars 2017, on recensait 6068 programmes BI proposés à travers le monde, dans 4655 écoles différentes.

4. Voir note 2. 


\section{LES CONFÉRENCES DE SÈVRES EN 1967 ET 1974}

La première période d'essor et de développement du BI, entre 1962 et 1974, est une période dite de "libéralisme intégré ", symbolisée par " la création et l'expérimentation"( Tarc, 2009). Au cours de cette période, tout semble en effet indiquer que le programme du diplôme du BI n'a à aucun moment été pensé comme un programme de masse, mais plutôt comme un projet élaboré dans des conditions expérimentales au sein des laboratoires que sont les «écoles internationales » indépendantes. L'idée était qu'une fois que ce programme aurait fait ses preuves, il pourrait ensuite être proposé aux concepteurs des curricula nationaux en tant que programme d'études testé pouvant être modifié et adapté aux contextes; selon Peterson (1972), les «méthodes expérimentales mises au point pour créer le baccalauréat international peuvent apporter des éléments utiles aux systèmes éducatifs nationaux ».

Au sein de ce processus, deux conférences organisées par le Centre international d'études pédagogiques (CIEP) à Sèvres ont joué un rôle majeur. L'élan initial en avait été donné par l'un des principaux bailleurs du projet, la Fondation Ford, « qui avait appuyé l'idée d'une conférence internationale majeure, durant laquelle les propositions de BI pourraient être présentées, et Madame Hatinguais [la directrice du centre] avait offert d'accueillir cet évènement à Sèvres " (Peterson, 1987). Le premier évènement, qualifié de "conférence majeure d'enseignants » (Peterson, 1972), s'est tenu à Sèvres en février 1967 et a rassemblé des experts venus de douze pays: Allemagne (de l'Ouest), Belgique, Bulgarie, Cameroun, États-Unis, France, Grande-Bretagne, Inde, Pologne, Suède, Suisse et Tanzanie. Cette conférence a indéniablement joué un rôle symbolique important, notamment sur le plan académique, et elle a également contribué à faire évoluer la perception du BI, jusqu'alors considéré comme un projet anglo-américain ; c'est à ce moment-là, véritablement, que le BI « a cessé d'être une vision vague et lointaine pour prendre les apparences d'un projet concret» (Peterson, 1972). Lors de cette conférence, les participants ont débattu du "schéma général de l'examen proposé »(Peterson, 1972). Cinquante années se sont écoulées depuis cet évènement majeur. Le principal objectif était initialement d'échanger sur les progrès accomplis jusqu'alors afin de promouvoir le BI (Hill, 2010). Mais cet objectif a été largement dépassé et des décisions importantes ont été prises concernant le profil à donner au diplôme du BI, par exemple en termes de notation des matières (de la note 1 à la note 7), dont une grande partie est toujours en vigueur de nos jours.

Selon Peterson (1972), « deux questions de la plus haute importance ont été débattues et réglées lors de cette conférence ». En premier lieu, l'examen devait-il être fondé sur un critère d'échec ou de réussite ? Il a été convenu à Sèvres que chaque matière recevrait une note, dans la mesure où " il était également possible que des pays du Tiers monde puissent vouloir utiliser cet examen 
à d'autres fins que celle de l'entrée dans les universités étrangères les plus sélectives»(Peterson, 1972). Ensuite, les élèves devaient-ils suivre le diplôme dans son intégralité (six matières) ? Il a au contraire été décidé que les élèves pourraient ne suivre que certains "certificats ». L'objectif poursuivi était que le BI n'apparaisse pas comme trop élitiste et de montrer qu'au contraire il « répondait aux besoins des élèves moins aptes scolairement, qui ne recherchent pas forcément l'entrée dans des universités très sélectives »(Peterson, 1972), tout en permettant en outre à des jeunes aux niveaux de compétences différents de travailler ensemble dans le cadre de leur scolarité. À la fin de la conférence, il a également été décidé d'organiser des examens expérimentaux de 1967 à 1970 , suivis d'une période expérimentale jusqu'en 1975. Le nombre de candidats serait ainsi limité à un échantillon de 500 élèves maximum chaque année. Surtout, cette conférence a donné " au BI un mandat pour aller de l'avant jusqu'en 1975 » (Hill, 2010) - date qui sera finalement repoussée à 1976 - et, à l'issue de cet évènement de trois jours, les participants sont repartis "vivement stimulés et avec un mandat clair»(Peterson, 1972). Cette conférence a indéniablement représenté un grand pas en avant et elle a permis au projet de " passer à un stade supérieur»(Hill, 2010).

Lors de la conférence de 1967 à Sèvres, il a également été décidé d’organiser une seconde conférence en 1974, à Sèvres également, afin d' " examiner les progrès accomplis au cours de la période expérimentale»(Hill, 2010). Du 22 au 26 avril 1974, cette conférence a réuni 64 participants de 21 pays. Elle a été financée par le ministère français de l'éducation. Il existait alors 23 écoles impliquées dans le "projet» de BI et la conférence a en outre permis de mettre en évidence le fait que 400 élèves du programme du diplôme du BI avaient été admis dans 200 universités de 25 pays différents (Hill, 2010). Si aucune décision majeure n'a été prise lors de cette conférence, elle a été l'occasion de formuler des conseils et des recommandations majeures; notamment, celle de faire en sorte que l' " expérimentation » devienne une opération permanente et que le BI puisse être proposé «dans le plus grand nombre de pays », représentant la plus grande variété possible de cultures et de traditions, « en particulier dans les pays en développement» (Hill, 2010).

Ce dernier point atteste de la préoccupation historique pour le déploiement géographique du BI. L'un des architectes majeurs du BI, déjà fréquemment cité, est l'Écossais Alec Peterson (1908-1988), que l'on a même qualifié de «père spirituel du BI»(voir Hill, 2010). En tant que directeur du département d'éducation de l'Université d'Oxford de 1958 à 1973, il a été un fervent détracteur de l'étroitesse et de la spécialisation excessive des examens anglais A Levels ${ }^{5}$ et

5. Les élèves anglais âgés de plus de 16 ans ont la possibilité de continuer dans un établissement secondaire pour préparer pendant deux ans l'examen du A-level, en vue d'entrer à l'université. En 2000, le contrôle continu a été introduit pour certaines matières ainsi qu'un système modulaire augmentant le nombre de disciplines à étudier. $(N d I R)$ 
il a également aidé l'Atlantic College à mettre au point un nouveau curriculum, plus vaste. Il est devenu le premier directeur général du BI en 1968 (et l'est resté jusqu'en 1977). Vers la fin de cette "première période », Peterson (1972) déplorait le peu d'activité en Afrique, qui était selon lui l'une des «deux principales déceptions dans le développement du projet jusqu'à présent»; la seconde était liée aux relations avec les anciens "pays socialistes». Il avait tout d'abord semblé assez confiant quant au rôle que pouvait jouer le BI en Afrique : "initialement, il avait semblé envisageable que la mise en place d'un programme d'études et d'un examen reconnus internationalement puisse être utile aux pays du Tiers monde ». Un tel programme pouvait, selon lui, permettre de "proposer un examen plus adapté aux besoins du continent africain » et " exempt de toute connotation coloniale »(Peterson, 1972).

\section{De SÈvRes À l’ÉquATEUR}

La période de croissance et de développement comprise entre 1974 et 1989 est considérée comme une période "transitoire ", caractérisée par l'émergence du BI en tant qu'institution éducative reconnue. Suite à la conférence de Sèvres en 1974, le BI s'est tout d'abord développé lentement. En 1977, seules 44 écoles proposaient le programme du diplôme du BI dans 18 pays. Le nombre d'écoles du BI a par la suite atteint 150 en 1982, et 700 en 1997, ce qui témoigne d'une croissance graduelle durant les années 1990 (Bunnell, 2016).

La période suivante, de 1990 à 2001, est, selon Tarc (2009), celle de l' " avènement de la mondialisation néolibérale », caractérisée par la " corporatisation» du BI. À la fin des années 1990 en effet, trois évolutions de cet ordre ont eu lieu. Tout d'abord, l'organisation du BI a amorcé un processus délibéré consistant à s'éloigner de sa base fondatrice, la communauté des " écoles internationales ", qu'elle commençait à considérer comme un "club exclusif », pour se tourner vers les écoles publiques nationales moins élitistes. En témoigne l'essor soudain des programmes du BI dans des régions comme Chicago. En second lieu, l'organisation du BI a commencé à se soucier de l'absence de planification de son processus de croissance. En 1999, une première tentative de formulation d'un processus de planification stratégique a été lancée, principalement axé sur le rôle de la technologie. En 2004, le processus de planification stratégique s'est fait plus décisif, avec comme priorités " une plus grande accessibilité » et "une croissance planifiée ». Une nouvelle rhétorique, résolument optimiste, est alors apparue, symbolisée par l'emploi de termes comme « impact accru » et "influence accrue » - sans nul doute le langage d'un BI plus sûr de lui et plus mûr, souhaitant exercer une plus grande influence. Enfin, troisième évolution, l'organisation du BI a commencé à se développer délibérément "à travers » des organisations, des réseaux et des gouvernements, plutôt que d'attendre que les écoles la contactent. Il s'agissait désormais de créer des «liens" plutôt que d'établir des «contacts» (Bunnell, 2008). 
La période depuis 2002 a été conceptualisée par Tarc (2009) comme étant la «quatrième période » de croissance et de développement historique du $\mathrm{BI}$, symbolisée par les tendances « de l'ère contemporaine " comme le branding 6 et la planification d'entreprise. Au niveau pédagogique, la formulation en 2007 des dix caractéristiques du "profil de l'apprenant BI » a contribué à renforcer la marque «BI». De même, l'émergence des quatre programmes du BI, en tant que "produits » testés internationalement et bénéficiant d'une garantie qualité, a probablement renforcé son attrait, en particulier auprès de gouvernements en quête d'un avantage compétitif dans un monde globalisé. Preuve en est le soutien inattendu apporté en 2006 au programme du diplôme du BI en Équateur par le président quasi-marxiste Rafael Correa ; en 2017, l'Équateur possède le troisième contingent d'écoles BI du monde (après les États-Unis et le Canada).

Ainsi, la décennie passée a vu le BI se développer avec plus de détermination, de confiance en soi et une identité plus affirmée, tant au niveau commercial que pédagogique. Le BI a considérablement évolué pendant cette période ; entre 2012 et 2017, le nombre de programmes du BI proposés à travers le monde a crû de près de 40 \%. Mais dans le même temps, des tensions et des critiques nouvelles sont apparues, comme nous le verrons dans la prochaine section.

\section{LE BI DANS SON CONTEXTE MODERNE FRAGMENTÉ}

Selon un cadre d'analyse conceptuel bien établi (Tarc, 2009), l'histoire et le développement du BI depuis 1962 comportent un ensemble de «tensions persistantes », principalement autour de trois dimensions : la citoyenneté, les objectifs curriculaires et le fonctionnement du BI (Tarc, 2009). Mais ce cadre d'analyse n'est sans doute pas exhaustif et d'autres "tensions " semblent avoir toujours existé. C'est le cas par exemple de la tension "géographique », qui peut également être perçue, en pratique, comme une tension de "déséquilibre ». Cette tension était évidente lors de la conférence de Sèvres en 1974 et elle avait été exprimée dès 1972 par Peterson.

En mars 2017, 61\% de l'ensemble des écoles BI du monde se trouvent dans la région des Amériques. En outre, $39 \%$ étaient situées aux États-Unis, où elles sont à $90 \%$ des écoles publiques. Les chiffres pour la session d'examen du diplôme du BI de mai 2016 indiquent que $54 \%$ des candidats (soit 81000 élèves) ont passé cet examen dans des écoles situées aux États-Unis. Sans nul doute, le BI a trouvé son "foyer» dans les écoles publiques états-uniennes, et il existe de nos jours un déséquilibre définitif de l'activité à l'échelle mondiale, suite à une longue période d'inactivité relative (Bunnell, 2011). Vient ensuite le Canada, avec $8 \%$ des écoles BI ; puis, en troisième position, comme nous l'avons déjà évoqué,

6. Le terme désigne une stratégie de marque ou institutionnelle. 
l'Équateur, avec 264 écoles, dont 204 sont subventionnées par l'État ${ }^{7}$. Ensuite, les quatre ensembles les plus importants sont situés en Australie, en Inde, au Royaume-Uni et en Chine. Au total, ces sept pays représentent en 2017 près des deux tiers de l'ensemble des écoles BI du monde. La France, quant à elle, ne dispose que de 17 écoles BI et leur croissance stagne depuis les années 1990.

Signalons également que les chiffres de la session d'examen de mai 2016 indiquent que près de $30 \%$ des écoles ont inscrit moins de vingt candidats aux épreuves, et $12 \%$ moins de dix élèves. Un nombre important d'écoles semble donc inscrire uniquement leurs meilleurs élèves et non l'ensemble d'une classe d'âge. Les données issues de la session de novembre 2016 (qui concernent principalement des pays du "Sud ») indiquent un nombre moyen d'inscriptions par école de seulement 14 candidats. Cette problématique, qui souligne la fragmentation existant au sein des écoles du BI, alarmerait les fondateurs ayant participé à la conférence de Sèvres en 1967, qui avaient souhaité une plus grande variété d'élèves, de tous niveaux scolaires. Mais le BI n'atteint en fait qu'un nombre limité d'élèves par école.

À l'autre extrémité, l'Afrique demeure en grande partie un désert en termes de BI, avec un déficit d'activité qui n'est pas sans rappeler les années 1980. C'est comme si le BI évoluait dans une "faille temporelle " en Afrique, représenté seulement par l'activité isolée des écoles internationales traditionnelles (Bunnell, 2016). Il n'y existe en effet aucune école BI nationale ou publique. On recense 23 écoles BI en Afrique du Nord et 54 dans toute l'Afrique subsaharienne. L'activité est également nettement insuffisante en Afrique francophone. Seules 8 écoles BI ont pu être recensées mi-février 2015 dans cinq des pays francophones de la région (Bunnell, 2016).

Le déséquilibre géographique du BI est donc évident et serait un sujet d'inquiétude majeur pour ses fondateurs, qui avaient souhaité une répartition géographique équilibrée. Ils ne souhaitaient en tout cas certainement pas que le BI ait un biais anglo-américain, comme l'a démontré la conférence de Sèvres. Si l'on met de côté l'anomalie équatorienne, le BI continue en 2017 sa croissance impressionnante dans les pays à haut revenu du "Nord », en particulier en Amérique du Nord. En 2017, 25 \% seulement de l'activité du BI a lieu dans les pays du Sud (tels qu'identifiés par l'appartenance au "Groupe des 77 » des Nations Unies). D’autre part, 70 \% de l'activité du BI a désormais lieu dans les pays plus développés du Nord (tels qu'identifiés par leur appartenance à l'Organisation de coopération et de développement économiques, OCDE). En particulier, trois régions du monde semblent défier la pénétration : le Moyen Orient, l'Afrique (Bunnell, 2016) et les nouveaux États indépendants de l'ère postsocialiste - ce qui n'est pas sans faire écho au commentaire sur les « deux déceptions » de Peterson en 1972. En parallèle, le BI poursuit son essor dans des endroits inattendus, comme au Pérou ou en Équateur.

7. Pour plus de détails sur l'activité du BI en Équateur, voir un rapport de Barnett (2013) disponible sur le site ibo.org. 
Le développement des programmes du BI implique également d'autres «tensions persistantes » (Tarc, 2009), aux implications politiques et sociales plus complexes. L'entrée du BI dans le secteur financé par les contribuables aux ÉtatsUnis, en 2003, a entraîné une critique virulente de la part des ultra-conservateurs (ou paléo-conservateurs, comme il est plus approprié de les appeler). Le BI a été accusé d'être "non américain » et considéré comme un complot émanant des Nations unies et de Genève destiné à promouvoir les valeurs universelles plutôt que les valeurs américaines (Bunnell, 2009). Un problème de fragmentation similaire s'est produit en Angleterre, où le BI a été perçu comme entrant en concurrence directe avec les controversés A-Levels, qui connaissaient depuis un certain nombre d'années une importante inflation de notes. En 2010, la présence du BI a culminé à 230 écoles en Angleterre mais a enregistré depuis un net déclin et l'on ne compte en 2017 plus que 130 écoles BI en Angleterre. Qui plus est, l'implantation du BI a évolué dans le pays : le BI est de plus en plus basé dans les écoles privées d'élite, tandis que les écoles publiques abandonnent ce qui est perçu comme un programme d'étude coûteux, ajoutant encore à la fragmentation de l'offre éducative entre secteur public et secteur indépendant en Angleterre (Bunnell, 2015).

En Australie, le programme du diplôme du BI a suscité beaucoup d'intérêt à la fois dans les écoles publiques et privées et a même été considéré comme un modèle possible pour le nouveau certificat d'éducation australien. Mais le développement du BI a également suscité des inquiétudes et des analyses sociologique critiques. Le BI est notamment apparu comme un choix clivant pour les parents :

l'émergence de ce programme d'études comme un programme d'élection par certains groupes semble aller à l'encontre de l'effort concomitant pour un curriculum national. (Doherty, 2009)

Le BI est ainsi devenu une force de fragmentation, et non d'unité, au sein de la société australienne car les parents rivalisent désormais entre eux pour obtenir un avantage compétitif; ainsi le BI « est impliqué non seulement dans des phénomènes de mobilité géographique, mais également dans des phénomènes de mobilité sociale et des stratégies de classe»(Doherty, 2009).

La morale de cette histoire est que le développement du BI n'est pas un jeu à somme nulle. Ses programmes coûteux sont souvent perçus comme détournant l'attention, le financement et les ressources des écoles publiques nationales. Si le BI se considère en théorie comme un catalyseur potentiel de développements curriculaires, il fonctionne souvent dans les faits comme une force élitiste clivante perturbant les systèmes nationaux. Il sera intéressant d'observer dans le futur la façon dont la croissance du BI sera reçue des commentateurs, ainsi que le déploiement envisagé dans les écoles publiques japonaises et dans des zones politiquement controversées comme l'Arabie Saoudite. 


\section{BIBLIOGRAPHIE}

BUNNELL T. (2016): "The dearth of International Baccalaureate Schools across Africa », Africa Education Review, n 13(2), p. 181-195.

BUNNELL T. (2015) : "The rise and decline of the IBDP in the United Kingdom », Oxford Review of Education, no 41(3), p. 387-403.

BUNNELL T. (2011) : "The International Baccalaureate in the United States: from relative inactivity to imbalance », The Educational Forum, n $75(1)$, p. 66-79.

BUNNELL T. (2009) : "The International Baccalaureate in the United States and the emerging 'culture war' ", Discourse: Studies in the Cultural Politics of Education, $\mathrm{n}^{\circ} 30(1)$, p. 61-72.

BUNNELL T. (2008) : « The global growth of the International Baccalaureate Diploma Programme over the first 40 years: a critical assessment ", Comparative Education, $\mathrm{n}^{\circ} 44(4)$, p. $409-424$.

DOHERTY C. (2009) : "The appeal of the International Baccalaureate in Australia's educational market: A curriculum of choice for mobile futures ", Discourse: Studies in the Cultural Politics of Education, $\mathrm{n}^{\circ}$ 30(1), p. 73-89.

HILL I. (2010) : The International Baccalaureate: Pioneering in Education (The International Schools Journal Compendium, Volume IV), John Catt : Woodbridge, Suffolk.

PETERSON A. (1972): The International Baccalaureate: An experiment in international education, Londres: George G. Harper \& Co. Ltd.

PETERSON A. (1987) : Schools Across Frontiers: The Story of the International Baccalaureate and the United World Colleges, La Salle, Illinois : Open Court.

TARC P. (2009): "What is the 'International' in the International Baccalaureate?

Three structuring tensions of the early years (1962-1973)», Journal of Research in International Education, $\mathrm{n}^{\circ}$ 8(3), p. 235-261. 
